\title{
Remedy: Supporting Consumer-Centered Medication Information Search
}

\author{
Lauren Wilcox, Steven Feiner \\ Columbia University \\ Department of Computer Science \\ New York, NY 10027 USA \\ \{wilcox,feiner\}@cs.columbia.edu
}

\author{
Noémie Elhadad, David Vawdrey \\ Columbia University \\ Department of Biomedical Informatics \\ New York, NY 10032 USA \\ noemie@dbmi.columbia.edu \\ david.vawdrey@dbmi.columbia.edu
}

\author{
Tran H. Tran \\ NewYork-Presbyterian Hospital \\ New York, NY 10032 USA \\ tht9016@nyp.org
}

\begin{abstract}
Remedy is a prototype consumer-centered medication-information search system, developed by applying textprocessing techniques and probabilistic generative topicmodeling approaches. Our prototype system includes tools to increase consumer recognition of quality and relevance indicators in medication-related search results. Attendees will be able to use Remedy to search for a range of over-the-counter and prescription medications, and can compare the search experience with that of popular online web search interfaces.
\end{abstract}

Keywords-WWW, health information search, health sensemaking

\section{INTRODUCTION}

Consumers are increasingly turning to popular search engine results to find websites describing symptoms, medical test results, and medications [9]. While searching for health-related information, consumers visit websites created by a variety of institutions and companies - from international online pharmacies, to personal blogs. The quality and accessibility of online materials is highly variable, even among the top sites returned by popular search engines [10]. In addition to search engine ranking, consumers often rely on the graphical design characteristics of a site to determine its credibility [4]. Unfortunately, this can be misleading [11]. Tools that aid consumers in reliably evaluating both the quality and relevance of health-related websites are currently missing from the web search user experience. To address this problem, we developed Remedy, a search prototype, shown in Fig. 1, that supports rapid filtering and comparison of medication-information search results, based on a number of website features and content topics.

\section{RELATED WORK}

Studies have examined the quality of online health information [5], and guidelines have been developed to assist end users in assessing health information quality [6,8]. Unfortunately, the use of guidelines is limited to those who know how to access and apply them and have the time to do so. Tools that directly support assessment of medication-information search results, during the search process itself, could provide necessary aids to help consumers navigate to relevant, high-quality sources.

Schwarz and Morris recently explored how credibility signals can be used to assist end users in evaluating website search results, for a number of information domains [10]. Online health information search can involve browsing multiple web pages to compare explanations, descriptions or opinions about a single topic. To assist end users with general sensemaking tasks, researchers have developed technologies that combine document summarization techniques with presentation techniques. Dontcheva et al. designed tools to allow end users to create and manage their own tabular document summaries created from website-specific extraction patterns [2]. Cheng and Gotz presented browser technology that recommends web document content, at the page-unit level, to end users to assist them in finding relationships among web pages [1]. In contrast, we focus on determining and presenting salient topic information, with the specific goal of augmenting the medication-information search experience. Remedy supports end-user assessment of website credibility, using metrics tailored to address medication-information search.

\section{PRototype}

To design Remedy, we drew on insights presented in the related work described above, as well as a literature review of consumer health-information search practices and patient information needs $[3,9,12,13]$. From this literature review, we identified four high-level principles that affect consumers' valuation of online health information: credibility, readability, consumer perspective, and topical relevance. These principles were embodied in Remedy through the design of the following search tools demonstrated in the video:

\section{A. Result-specific topic map}

For each web page result, a set of horizontal bars (shown in Fig. 1), indicates the relative amount of page content for each of the set of topics, as determined by our topic analysis. Selecting a topic in the map provides "pop-up snippets", which preview related excerpts from the page.

\section{B. Result-specific feature annotations}

Certain binary characteristics are displayed in the bulleted list for each web page result, as shown in Fig. 1. Features include whether the page was reviewed by experts, contains commercial content, or incorporates patient reviews. 


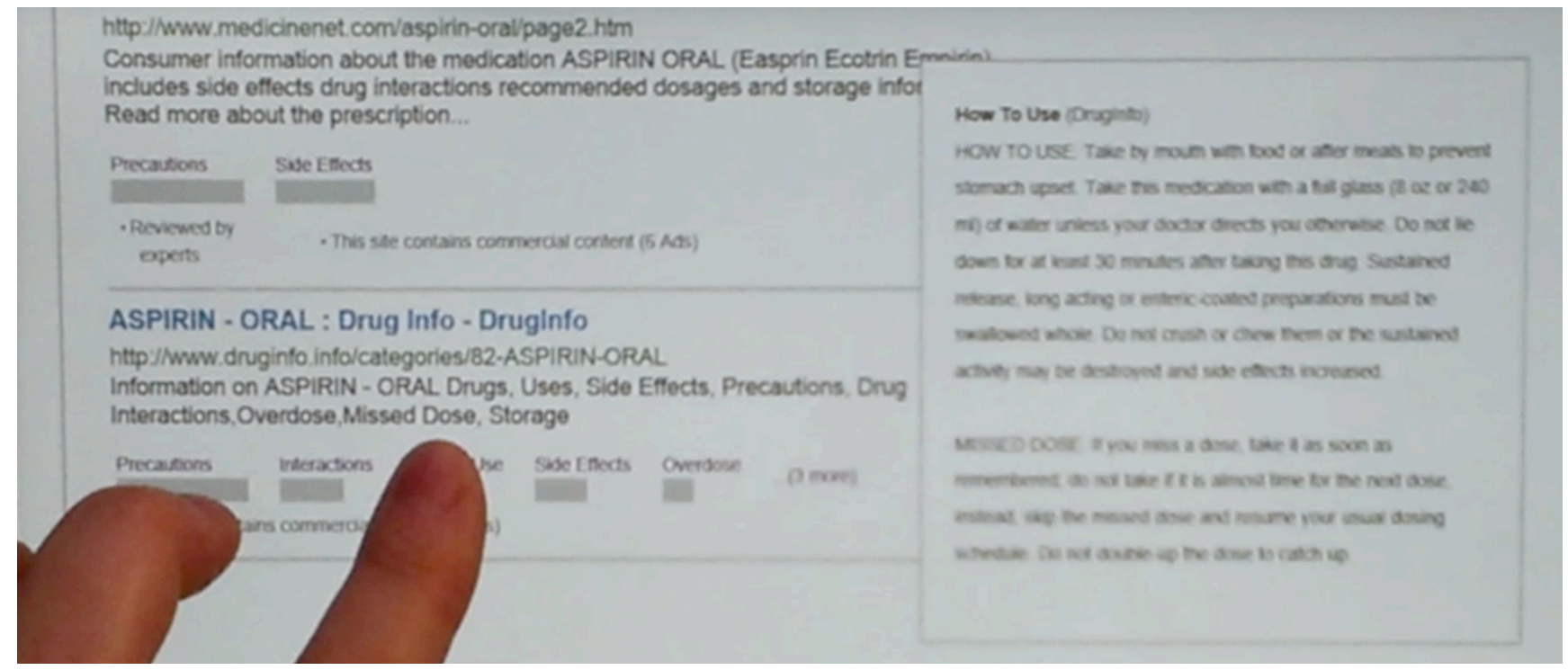

Fig. 1. User explores search results for "Aspirin" returned by Remedy. Touching a topic in the topic map for a result shows topic-specific "pop-up snippets" from the linked web resource (on the right). These snippets are determined by topical relevance, in addition to keyword-based metrics. Here, the user touches "How to Use" to see relevant snippets from www.druginfo.info.

\section{Topic Menu: browse all results by topic}

Selecting a topic (e.g., "Dose" or "How to Use") from the topic menu will display topically-relevant page content (as "inline snippets", shown in the video), collected across all page results for the queried medication.

\section{Filters to select results based on page features}

Selecting one or more filter criteria narrows the search results to ones that satisfy all selected criteria.

These tools provide web page features not available to consumers today. To develop Remedy, we combined topicmodeling techniques with presentation and interaction techniques, to support topic-based browsing of information across medication information web pages. We applied LDA-based topic modeling algorithms [7] to generate topic clusters, along with additional text processing and manual content curation. The search interface was created using HTML with JavaScript and PHP. A database hosted on a secure web server stores all metadata and indexing information.

\section{DEMO}

Using slate computers, attendees will be able to search for a range of over-the-counter and prescription medications and compare Remedy with popular online web search interfaces. We expect the demo to appeal not only to researchers working on medication search in particular, but to researchers interested in domain-specific search for other health-related fields.

\section{ACKNOWLEDGMENTS}

This research was supported in part by AHRQ Grant 1R36HS021393 and a gift from Microsoft Research.

\section{REFERENCES}

[1] Cheng W, Gotz D. "Context-based page unit recommendation for webbased sensemaking tasks." Proc ACM IUI 2009, 107-116.

[2] Dontcheva M, Drucker SM, Salesin D, Cohen MF. "Relations, cards, and search templates: User-guided web data integration and layout." Proc ACM UIST 2007. ACM, New York, NY, USA, 61-70.

[3] Eysenbach G, Köhler C. "How do consumers search for and appraise health information on the world wide web?" BMJ. 2002 Mar 9;324 (7337):573-7.

[4] Fogg BJ, Soohoo C, Danielson DR, Marable L, Stanford J, Tauber ER. "How do users evaluate the credibility of Web sites? A study with over 2,500 participants." Proc DUX 2003, 1-15.

[5] Jadad A, Gagliardi A. "Rating health information on the Internet: Navigating to knowledge or to Babel?" J Am Medical Assoc 1998;279:611-4. “

[6] Library of Congress. "Locating health and medical information." Science reference guide no. 34. August 2010. Retrieved March 3, 2013. http://www.loc.gov/rr/scitech/SciRefGuides/medicalinfo.html\#safe

[7] McCallum AK. "MALLET: MAchine Learning for LanguagE Toolkit." http://mallet.cs.umass.edu. 2002.

[8] MedlinePlus Quality Guidelines. Retrieved March 3, 2013. http://www.nlm.nih.gov/medlineplus/criteria.html

[9] PwC HRI Social Media Consumer Survey, 2012. Retrieved March 3, 2013. http://www.pwc.com/us/en/health-industries/publications/healthcare-social-media.jhtml

[10] Schwarz J, Morris M. "Augmenting web pages and search results to support credibility assessment." Proc CHI 2011. ACM, New York, NY, USA, 1245-1254.

[11] Sillence E, Briggs P, Harris PR, Fishwick L. "How do patients evaluate and make use of online health information?" Social Science \& Medicine 64 (2007) 1853-1862.

[12] Slater MD, Zimmerman DE. "Descriptions of web sites in search listings: A potential obstacle to informed choice of health information." Am J Public Health. 2003 Aug; 93(8):1281-2.

[13] Wang $\mathrm{L}$ et al. "Using internet search engines to obtain medical information: A comparative study." J Med Internet Res 2012;14(3):e74. 